\title{
VECTOR LATTICES OVER SUBFIELDS OF THE REALS
}

\author{
P. BIXLER, P. CONRAD, W. B. POWELL and C. TSINAKIS
}

(Received 9 September 1987)

Communicated by $\mathbf{H}$. Lausch

\begin{abstract}
In this paper we consider classes of vector lattices over subfields of the real numbers. Among other properties we relate the archimedean condition of such a vector lattice to the uniqueness of scalar multiplication and the linearity of $l$-automorphisms. If a vector lattice in the classes considered admits an essential subgroup that is not a minimal prime, then it also admits a non-linear $l$-automorphism and more than one scalar multiplication. It is also shown that each $l$-group contains a largest archimedean convex $l$-subgroup which admits a unique scalar multiplication.
\end{abstract}

1980 Mathematics subject classification (Amer. Math. Soc.) (1985 Revision): 06 F 20.

\section{Introduction}

Throughout this paper let $F$ be an ordered subfield of the real field $\mathbb{R}$, and let $V_{F}$ be the class of all vector lattices over $F$. Thus, $G \in V_{F}$ if $G$ is an abelian $l$-group and a vector space over $F$ such that $0<r \in f$ and $0<g \in G \Rightarrow 0<r g$. It is well-known that $V_{F}$ is closed with respect to $l$-homomorphic images, $l$-ideals, and cardinal products. In [11] Martinez asserts that $V_{\mathbb{R}}$ is closed with respect to joins of convex $l$-subgroups and hence is a torsion class of $l$-groups. Whether or not this is true is doubtful and also a very difficult question to answer. In this paper we find several interesting classes $S$ of $l$-groups so that $S \cap V_{F}$ is a torsion class.

We first consider the following properties of $G \in V_{F}$ with $F \neq \mathbb{Q}$, the rational field.

(1) $G$ is archimedean.

(C) 1990 Australian Mathematical Society $0263-6115 / 90 \$ A 2.00+0.00$ 
(2) The scalar multiplication on $G$ is unique.

(3) Each $l$-automorphism of $G$ is linear with respect to $F$.

We will see that $(1) \Rightarrow(2) \Rightarrow(3)$, but whether or not $(2) \Rightarrow(1),(3) \Rightarrow(1)$, or $(3) \Rightarrow(2)$ is an open question.

We prove that if $G$ has an essential subgroup that is not a minimal prime, then it admits an $l$-automorphism that is not linear and so it has at least two scalar multiplications. There are several consequences of these results. If $\mathrm{Ar}$ is the class of archimedean $l$-groups, then $\operatorname{Ar} \cap V_{F}$ is closed with respect to convex $l$-subgroups, joins of convex $l$-subgroups, and images of complete $l$-homomorphisms. Hence $A r \cap V_{F}$ is a pseudo-torsion class. In particular, each $l$-group contains a largest archimedean convex $l$-subgroup that admits a unique scalar multiplication by elements of $F$. Each archimedean $l$-group contains a largest $l$-subgroup that belongs to $V_{F}$. It follows that an archimedean $l$-group $G$ "knows" whether or not it belongs to $V_{F}$. For example, $G \in V_{F}$ if and only if each maximal $o$-subgroup of $G$ belongs to $V_{F}$. Also $G \in V_{\mathbf{R}}$ if and only if each maximal $o$-subgroup of $G$ is $a$-closed.

We will show that for $G \in V_{\mathbb{R}}$ the following are equivalent:

(1) $G$ is archimedean.

(2) Each maximal archimedean $o$-subgroup is a subspace.

(3) Each $a$-closed $o$-subgroup is a subspace.

In Section 4 we show that each l-group "knows" whether or not it belongs to $V_{F}$.

For the class $A$ of abelian $l$-groups we consider the free product of vector lattices viewed as members of $A$. We have that the following properties of $F$ are equivalent:

(1) $F \sqcup F$ is archimedean.

(2) $F \sqcup F \in V_{F}$.

(3) $\bigsqcup G_{i} \in V_{F}$ for any family $\left(G_{i} \mid i \in I\right) \subseteq V_{F}$.

(4) $F=\mathbb{Q}$.

Finally, if $M$ is the torsion class of all $l$-groups such that their principal polars satisfy the DCC, then $V_{F} \cap M$ is a torsion class. Also, for an abelian l-group $G \in M$, we have $G \in V_{F}$ if and only if $G / P \in V_{F}$ for each minimal prime $P$.

Notation AND Definitions. If $G$ is an $l$-group, then we denote by $G^{d}$ its divisible hull. If $G$ is archimedean, then its Dedekind-MacNeille completion will be written $G^{\wedge}$. The cardinal sum of a family $\left(G_{i} \mid i \in I\right)$ of $l$-groups is denote by $\sum G_{i}$ while the cardinal product of this family is written $\Pi G_{i}$.

A partially ordered set $\Gamma$ is a root system if $\{\gamma \in \Gamma \mid \gamma \geq \alpha\}$ is a chain for each $\alpha \in \Gamma$. Let $V(\Gamma, F)$ be the set of all functions of $\Gamma$ into $F$ whose 
supports satisfy the ACC. A component $v_{y}$ of $v \in V=V(\Gamma, F)$ is maximal if $v_{\gamma} \neq 0$ and $v_{\alpha}=0$ for all $\alpha>\gamma$. Define $v \in V$ to be positive if each maximal component is positive. Then $V \in V_{F}$ and each group in $V_{F}$ can be embedded in such a $V$ (see [3] or [5]) for an appropriate choice of $\Gamma$. Let

$$
\Sigma=\Sigma(\Gamma, F)=\{v \in V \mid v \text { has finite support }\} .
$$

Then $\Sigma$ is an $l$-subgroup of $V$ and also a subspace.

For further information on terms and notation, the reader is referred to Conrad [5].

Let us begin by considering $G, H \in V_{F}$ with $H$ archimedean. Before we prove the uniqueness of scalar multiplication on $G$ we make the following two observations.

(A) Each $l$-homomorphism of $G$ into $H$ must be linear (for a proof see $[4$, p. 227]).

(B) If $\delta$ is an $l$-automorphism of $G$ that is not linear and we define

$$
r \Delta g=(r(g \delta)) \delta^{-1},
$$

then $\Delta$ is a new scalar multiplication for $G$ and $\delta$ is a linear $l$-isomorphism of $G$ onto $(G, \Delta)$.

Now consider the following properties of $G$.

(1) $G$ is archimedean.

(2) The scalar multiplication of $G$ is unique.

(3) Each $l$-automorphism of $G$ is linear with respect to $F$.

Note that if $F=\mathbb{Q}$, then (2) and (3) hold. The implications (1) $\Rightarrow(2) \Rightarrow(3)$ are established in [8], but for completeness we give a proof here.

$(1) \Rightarrow(2)$ : If $\circ$ and \# are scalar multiplications on $G$, then by (A) the identity map is a linear map of $(G, \circ)$ onto $(G, \#)$ so the multiplications must agree.

(2) $\Rightarrow(3)$ : This is an immediate consequence of (B).

THEOREM 2.1. If $G \in V_{F}$ with $F \neq \mathbb{Q}$ and if $G$ has an essential subgroup $G_{\lambda}$ that is not a minimal prime, then $G$ admits an l-automorphism that is not linear and hence $G$ admits at least two scalar multiplications.

Proof. Let $\Gamma(G)$ be the set of all pairs $\left(G^{\gamma}, G_{y}\right)$ of convex $l$-subgroups of $G$ such that $G_{\gamma}$ is maximal without some element of $G$ and $G^{\gamma}$ covers $G_{\gamma}$. 
Without loss of generality (see (3)) we may assume that $G$ is an $l$-subgroup and an $F$-subspace of $V=V(\Gamma(G), \mathbb{R})$. Now since $G_{\lambda}$ is essential and not minimal there exists an element $0<b \in G$ so that each value of $b$ is less than $\lambda$ and so that each maximal component of $b$ is less than $\lambda$.

Now let $\rho$ be the projection of the elements of $G$ onto the $\lambda$ th component. Note that $G \rho$ is a subgroup of $V$, but $G \rho$ need not be a subset of $G$. Let $\alpha$ be a group homomorphism of $G \rho$ into the subgroup $F b$ of $G$ that is not linear (here we use the hypothesis of $F \neq \mathbb{Q}$ ). Finally, for each $g \in G$ define $g \tau=g+g \rho \alpha$. Clearly $\tau$ is an endomorphism of $G$. Now $g \rho \alpha \in F b$ so its projection onto $\lambda$ is zero. Thus, $g \rho \alpha \rho=0$ so $(g-g \rho \alpha) \tau=g$ and hence $\tau$ is onto. If $0=g+g \rho \alpha$, then $0=g \rho+g \rho \alpha \rho=g \rho$ so $g=0$. Thus, $\tau$ is an automorphism of $G$.

If $g \rho \alpha \neq 0$, then $\lambda$ is contained in the support of $g$ so $|g|>n|g \rho \alpha|$ for all positive integers $n$. But this implies $g>0$ if and only if $g+g \rho \alpha>0$, and hence $\tau$ is an $l$-automorphism of $G$ that is not linear.

In particular, if $\Gamma$ is a root system that is not trivially ordered, then $V(\Gamma, F)$ and $\Sigma(\Gamma, F)$ have more than one scalar multiplication. Also, a non-archimedean completely distributive $G \in V_{F}$ has more than one scalar multiplication since $G$ has a representing system of essential subgroups.

We turn now to the problem of embedding abelian $l$-groups into vector lattices over $F$. To this end we say that $U$ is an $F$-hull of an abelian $l$-group $G$ if

(a) $U \in V_{F}$,

(b) $G$ is a large $l$-subgroup of $U$, and

(c) no proper $l$-subspace of $U$ contains $G$.

For the case where $F=\mathbb{R}$ the following four propositions have been proved in [6], [2], [7], and [8], respectively. Analogous proofs yield the corresponding results when $F$ is an arbitrary subfield of $\mathbb{R}$.

Proposition 2.2. Each abelian l-group admits an $F$-hull. If $G$ is an archimedean l-group, then $G$ admits a unique $F$-hull $G^{F}$. This $F$-hull is l-isomorphic to the l-subspace of the $F$-vector space $\left(G^{d}\right)^{\wedge}$ that is generated by $G$, and hence it is archimedean.

Proposition 2.3. If $G$ is archimedean, then $G^{F}$ is the smallest archimedean member of $V_{F}$ that contains $G$.

Proposition 2.4. If $G$ is an archimedean f-ring, then there exists a unique multiplication on $G^{F}$ making $G^{F}$ into an $f$-ring with $G$ as a subring. 
Proposition 2.5. Each archimedean l-group $G$ contains a largest l-subgroup $F(G)$ that belongs to $V_{F} . F(G)$ is the largest l-subspace of $G^{F}$ that is contained in $G$, and it is also a characteristic l-subgroup of $G$.

\section{3}

In this section we consider archimedean $l$-groups and their relationship to $V_{F}$. In particular, if $G$ is archimedean, then from Section 2 we have

$$
F(G) \subseteq G \subseteq G^{F} \subseteq\left(G^{d}\right)^{\wedge} .
$$

Note that $G \in V_{F}$ if and only if $G$ is an $F$-subspace of $\left(G^{d}\right)^{\wedge}$. Thus an archimedean $l$-group "knows" whether or not it belongs to $V_{F}$. Later we will get some nicer versions of this fact.

We now describe the $F$-space $F(G) \subseteq G$ whose existence is guaranteed in Proposition 2.5.

Proposition 3.1. If $G$ is an archimedean l-group, then

$$
F(G)=\{x \in G \mid F x \subseteq G\} .
$$

Proof. If $x \in F(G)$, then clearly $F x \subseteq G$. Conversely, suppose $x \in G$ and $F x \subseteq G$ (the product $F x$ is formed in $G^{F}$ ). For $0<a \in F$ we have $(a x)^{+}-(a x)^{-}=a\left(x^{+}\right)=a x \in G$ so $a\left(x^{+}\right)=(a x)^{+} \in G$. Thus, $F x^{+}$is an $l$-subgroup of $G$ that belongs to $V_{F}$ and $x^{+} \in F(G)$. Similarly, $x^{-} \in F(G)$ and thus $x \in F(G)$.

COROLlary 3.2. If $G$ is an archimedean $f$-ring, then $F(G)$ is a ring ideal of $G$.

Proof. If $x \in F(G)$ and $y \in G$, then $F x \subseteq G$ so $F(x y)=F(x) y \subseteq G$. Hence, $x y \in F(G)$.

Now let $A$ be an archimedean $o$-subgroup of $G \in V_{\mathbb{R}}$. We may assume that $G$ is an $l$-subspace of $V(\Gamma, \mathbb{R})$ (see [3]). Pick $0<a \in A$ and consider the set $\left\{a_{\delta} \mid \delta \in \Delta\right\}$ of the maximal components of $a$. Let $\rho$ be the projection of $V$ onto $\Delta$ and for each $\delta \in \Delta$ let $\rho \delta$ be the projection of $V$ onto $\delta$. Using this notation we establish the next important lemma.

Lemma 3.3. (1) $\rho$ and $\rho \delta$ induce o-isomorphisms on $A$ and $A \rho \subseteq$ $\mathbb{R}(a \rho)$

(2) $A$ is maximal if and only if $A \rho=\mathbb{R}(a \rho)$ if and only if $A$ is a-closed. 
(3) If $G$ is archimedean, then $A \subseteq \mathbb{R} a$, and $A=\mathbb{R} a$ if and only if $A$ is maximal.

(4) If $H$ is an o-subgroup of an archimedean l-group $K$ and $0<h \in H$, then $H \subseteq \mathbb{R} h$, the subspace of $K^{\mathbb{R}}$ determined by $h$.

Proof. (1) By using a suitable $l$-automorphism of $V$ we may assume each $a_{\delta}=1$. Now for $0<b \in A, n a>b$ and $n b>a$ for some $n>0$, so $\left\{b_{\delta} \mid \delta \in \Delta\right\}$ is the set of maximal components of $b$. It follows that for $x, y \in A$ we have

$$
\begin{array}{ll}
x<y & \text { if and only if } x_{\delta}<y_{\delta} \text { for all } \delta \in \Delta \\
& \text { if and only if } x_{\delta}<y_{\delta} \text { for some } \delta \in \Delta \text { and } \\
x=y & \text { if and only if } x_{\delta}=y_{\delta} \text { for all } \delta \in \Delta \\
& \text { if and only if } x_{\delta}=y_{\delta} \text { for some } \delta \in \Delta .
\end{array}
$$

Thus, $\rho$ and $\rho \delta$ induce $o$-isomorphisms on $A$. Now, consider $x \in A$ and $\alpha, \beta \in \Delta$. The map $x_{\alpha} \rightarrow x_{\beta}$ is an $o$-isomorphism so $x_{\beta}=k_{\beta} x_{\alpha}$ for some fixed $0<k_{\beta} \in \mathbb{R}$. But since $a_{\beta}=a_{\alpha}=1$ we have $k_{\beta}=1$ so $x_{\alpha}=x_{\beta}$ for all $\alpha, \beta \in \Delta$. Thus, $a \rho \subseteq \mathbb{R}(a \rho)$.

(2) Let $D=\{r \in \mathbb{R} \mid r(a \rho) \in A \rho\}=\left\{r \in \mathbb{R} \mid x_{\delta}=r\right.$ for some $\left.x \in A\right\} \cong A$. Now suppose $A$ is maximal. Then $A$ is divisible so $\mathbb{R}=D \oplus K$. By way of contradiction let us suppose $0<k \in K$. Then $k a \in G$ and so $A \oplus\langle k a\rangle$ is an archimedean $o$-subgroup of $G$ that properly contains $A$. This contradiction implies $K=0$ so $A \cong D=\mathbb{R}$ and $A \rho=\mathbb{R}(a \rho)$.

(3) Let $B$ be a maximal archimedean $o$-subgroup that contains $A$. Then as above we get $B \rho=\mathbb{R}(a \rho)$ so $\rho^{-1}$ is an $l$-isomorphism of the vector space $B \rho$ into the archimedean vector lattice $G$. This means $\rho^{-1}$ must be linear, and therefore $A \subseteq B=\mathbb{R} a$.

(4) $H$ is contained in a maximal $o$-subgroup $A$ of $K^{\mathbb{R}}$ so by (3) we have $H \subseteq A=\mathbb{R} h$.

We note that the proof of (1) is valid for $G \in V_{F}$.

Using Lemma 3.3 we are able to determine when certain $o$-subgroups are subspaces.

THEOREM 3.4. For $G \in V_{\mathbf{R}}$ the following are equivalent.

(1) $G$ is archimedean.

(2) Each maximal archimedean o-subgroup is a subspace.

(3) Each a-closed o-subgroup is a subspace.

Proof. $(1 \Rightarrow 2)$ This follows from (3) of Lemma 3.3.

$(1 \Rightarrow 3)$ Since each $a$-closed $o$-subgroup is a maximal archimedean $o$ subgroup this is a consequence of the preceding implication. 
( $2 \Rightarrow 1$ and $3 \Rightarrow 1$ ) Suppose $G$ is not archimedean. Then $0<b \ll a$ for some $a, b \in G$. Now, $\mathbb{R}=\mathbb{Q} \oplus D$ so let $A=\mathbb{Q}(a+b)+D a \cong \mathbb{R}$. This is a maximal archimedean $o$-subgroup of $G$ which is $a$-closed, but it is not a subspace of $G$.

THEOREM 3.5. For an archimedean l-group $G$ the following are equivalent.

(1) $G \in V_{F}$.

(2) Each maximal o-subgroup $H$ belongs to $V_{F}$.

(3) Each $0<x \in G$ is contained in an o-subgroup $H$ where $H \in V_{F}$.

Proof. $(1 \Rightarrow 2)$ By (4) of Lemma 3.3 we get that $H \subseteq \mathbb{R} h$ and that $F h$ is an $O$-subgroup of $G$. Thus, $H+F h$ is contained in the $o$-group $\mathbb{R} h$. Since $H$ is a maximal $o$-subgroup of $G$ we have $F h \subseteq H$, and hence $H \in V_{F}$.

$(2 \Rightarrow 3)$ This is clearly true since each $x>0$ in $G$ is contained in a maximal $o$-subgroup $H$.

$(3 \Rightarrow 1)$ Since $F x \subseteq H \subseteq G$, we have $G=F(G) \in V_{F}$.

When $F=\mathbb{R}$ we get an even stronger version of Theorem 3.5.

THEOREM 3.6. For an archimedean l-group $G$ the following are equivalent.

(1) $G \in V_{\mathbf{R}}$.

(2) Each maximal o-subgroup is a-closed.

(3) Each $0<x \in G$ is contained in an o-subgroup that is a-closed.

(4) If $0<x \in G^{\gamma} \backslash G_{\gamma}$, then $G^{\gamma}=G_{\gamma} \oplus D_{\gamma}$ where $D$ is an a-closed o-group that contains $x$.

Proof. Since an archimedean $o$-group $H$ is $a$-closed if and only if $H=$ $\mathbb{R}$ we see that (1), (2), and (3) are equivalent. Also, it is clear that (4) implies (3).

$(1 \Rightarrow 4)$ We have $G^{\gamma} \supseteq G_{\gamma} \oplus \mathbb{R} x$ and $\mathbb{R} \cong\left(G_{\gamma} \oplus \mathbb{R} x\right) / G_{y} \subseteq G^{\gamma} / G_{\gamma} \cong \mathbb{R}$ so this gives an $o$-isomorphism of $\mathbb{R}$ into $\mathbb{R}$ which must be onto. Hence $G \oplus \mathbb{R} x=G^{\gamma}$.

In the above theorem the hypothesis that $G$ is archimedean cannot be removed. For example, consider $G=\sum_{i=1}^{\infty} \overrightarrow{\mathbb{R}}_{i} \oplus \mathbb{Z}(1,1, \ldots)$. Then $G$ satisfies (4) but not (1).

In this section we show that an $l$-group knows whether or not it is a vector lattice over $F$. We use the embedding theorem from [3] and some variations of this theory developed in [5]. Each group $V_{F}$ is divisible so we can restrict our attention to such a group $G$. If $\Delta$ is a plenary subset of $\Gamma(G)$, then there 
exists an embedding $\tau$ of $G$ into $V(\Delta, \mathbb{R})$ so that $g \in G^{\delta} \backslash G_{\delta}$ if and only if $(g \tau)_{\delta}$ is a maximal component of $g \tau$. Thus, we may assume $G \subseteq V(\Delta, \mathbb{R})$ and for each $\delta \in \Delta$ there is an element in $G$ with maximal component at $\delta$. Also, since $F \subseteq \mathbb{R}$ there is a natural scalar multiplication on $V$ so that it is a vector lattice over $F$.

An $\eta$-automorphism of $V$ is an $l$-automorphism that induces the identity on the maximal components of each element of $V$.

Proposition 4.1. $G \in V_{F}$ if and only if there exists an l-automorphism $\sigma$ of $V$ such that $G \sigma$ is an $F$-subspace of $V$.

Proof. It is clear that if the condition is satisfied then $G \in V_{F}$. Assume now that $G \in V_{F}$. By the embedding theorem there exists a linear $l$-isomorphism $\alpha$ of $G$ into $V$ so that $g \in G$ has a maximal component at $\delta$ if and only if $g \alpha$ has a maximal component at $\delta$. By following $\alpha$ with a suitable $l$-automorphism of $V$ we may assume this $\alpha$ induces the identity on the maximal components of the elements from $G$. Finally, these two embeddings are connected by an $l$-automorphism $\sigma$ of $V$, and since for each $\delta \in \Delta$ there is an element in $G$ with maximal component at $\delta$, it follows that $\sigma$ is an $\eta$-automorphism of $V$.

Now suppose that $G \in V_{F}$. We may assume without loss of generality that $G$ is an $F$-subspace of $V$.

CoROllary 4.2. Each scalar multiplication of $G$ by $F$ with $G \in V_{F}$ is determined by the $\eta$-automorphism $\sigma$ of $V$ so that $G \sigma$ is also a subspace of $V$. Here $r \# g=(r(g \sigma)) \sigma^{-1}$ is the new scalar multiplication.

Proof. This follows from Proposition 4.1. An alternate proof can be found in [8].

Now, let $\circ$ and \# be scalar multiplications for $G$ so that $(G, \circ)$ and $(G, \#)$ are vector lattices over $F$. It is an open question whether or not these scalar multiplications are connected by an $l$-automorphism of $G$. In fact it is not known whether $(G, o)$ and $(G, \#)$ have the same dimension. Once again the answer is related to the automorphism structure of $V$. We can assume from the above that $(G, 0)$ is a subspace of $V$ and that there exists an $\eta$-automorphism $\sigma$ of $V$ that induces a linear $l$-isomorphism of $(G, \#)$ into $V$.

$$
(G, \circ) \rightarrow V, \quad(G, \#) \stackrel{\sigma}{\longrightarrow} V .
$$

Proposition 4.3. The scalar multiplications $\circ$ and \# are connected by an l-automorphism of $G$ if and only if there exists a linear l-automorphism of $V$ such that $G \tau=G \sigma$. 
Proof. Suppose first that $\beta$ is an $l$-automorphism of $G$ with $(r \circ g) \beta=$ $r \# g \beta$ for all $r \in F$ and $g \in G$. Then $\beta \sigma$ is a linear $l$-isomorphism of $G$ onto $G \sigma$ and hence it can be lifted to a linear $l$-automorphism $\tau$ of $V$.

Conversely, suppose there exists a linear $l$-automorphism $\tau$ of $V$ such that $G \tau=G \sigma$. Then $\sigma \tau^{-1}$ induces an $l$-automrophism on $G$ and

$$
\left.(r \# g) \sigma \tau^{-1}=(r \circ(g \sigma)) \tau^{-1}\right)=r \circ\left(g \sigma t^{-1}\right) .
$$

Now, let $H$ be the class of all $F$-vector lattices such that any two scalar multiplications are connected by an $l$-automorphism. Also let $K$ be the class of all $F$-vector lattices $A$ such that if $B \in V_{F}$ with $A \cong B$ as $l$-groups, then they are isomorphic as $F$-vector lattices.

It is easy to show that $H=K$ and $H$ is closed with respect to cardinal sums and products. In particular the following are equivalent.

(1) Any two scalar multiplications for an $F$-vector lattice are connected by an $l$-automrophism.

(2) If two $F$-vector lattices are isomorphic as $l$-groups, then they are isomorphic as $F$-vector lattices.

In this section we investigate the relationship between $V_{F}$ and the free product of abelian $l$-groups. If $G$ and $H$ are abelian $l$-groups, then $G \sqcup H$ will denote their abelian $l$-group free product. Hence, $G \sqcup H$ is an abelian $l$-group and each pair of $l$-homomorphisms of $G$ and $H$ into an abelian $l$-group $K$ can be extended to an $l$-homomorphism of $G \sqcup H$ into $K$.

Let $A$ and $B$ be subgroups of $\mathbb{R}$. Then $(a, b),(c, d) \in A \boxplus B$ are separated if $(a, b)+r(c, d)=0$ for some $0<r \in \mathbb{R}$, and they are positively independent if $m(a, b)+n(c, d) \leq 0$ for $0 \leq m, n \in \mathbb{Z}$ implies $m=n=0$.

TheOREM 4.1 (Martinez [10]). For subgroups $A$ and $B$ of $\mathbb{R}$, the following are equivalent:

(1) $A \sqcup B$ is a subdirect product of copies of $\mathbb{R}$.

(2) $A \cup B$ is archimedean.

(3) $A \boxplus B$ contains no separated positively independent pair.

The next proposition gives a condition that is a bit more informative and easier to check than (3).

Proposition 5.2. For subgroups $A$ and $B$ of $\mathbb{R}$, the following are equivalent. 
(1) $A \sqcup B$ is not archimedean.

(2) $x A \cap B$ has rank $>1$ for some $0<x \in \mathbb{R}$.

Proof. Assume first that $A \sqcup B$ is not archimedean and let $(a, b)$ and $(c, d)$ be a separated, positively independent pair from $A \boxplus B$. Thus, for some $0<r \in \mathbb{R}$ we have $(a, b)+r(c, d)=0$. If $r$ is rational, then $(a, b)$ and $(c, d)$ are not positively independent so $r$ must be irrational. Now, $a+r c=0$ in $A$ and $b+r d=0$ in $B$. If $c=0$, then $a=0$. But then $(0, b)$ and $(0, d)$ are not positively independent; for either $b=d=0$ or $b d<0$ so $m b+n d \leq 0$ for $0<m, n \in \mathbb{Z}$. Thus, $c \neq 0$ and similarly $d \neq 0$.

Now, multiply $A$ by $1 /|c|$ and get $a /|c|+r( \pm 1)=0$. Then $r, 1 \in$ $(1 /|c|) A \cap(1 /|d|) B$. Hence, $(1 /|c|) A \cap(1 /|d|) B$ has rank $>1$ and so also does $|d / c| A \cap B$.

Conversely, assume that there exists $0<x \in \mathbb{R}$ such that $x A \cap B$ has rank $>1$. Pick $0<y \in x A \cap B$. Then $1 \in y^{-1}(x A \cap B)=y^{-1} x A \cap y^{-1} B$ and $y^{-1} x A \sqcup y^{-1} B \cong A \sqcup B$. So without loss of generality we have 1 , $t \in A \cap B$ with $0<t$ irrational and $(t,-t)+t(-1,1)=0$. Suppose that $m(t,-t)+n(-1,1) \leq 0$. Then $m t-n \leq 0$ and $-m t+n \leq 0$ so $m t-n=0$ and hence $m=n=0$. Thus, $(t,-t)$ and $(-1,-1)$ is a separated, positively independent pair from $A \boxplus B$ and $A \sqcup B$ cannot be archimedean.

Several corollaries are immediate from Proposition 5.2.

COROLlary 5.3. $A \cup B$ is archimedean if and only if $x A \cap B$ has rank 1 for all $0<x \in \mathbb{R}$.

Corollary 5.4. $A \sqcup A$ is archimedean if and only if $A$ has rank 1.

COROLlary 5.5. If $A$ has rank 1 , then $A \sqcup B$ is archimedean.

Proposition 5.6. If $H$ is a divisible abelian subgroup of an l-group $G$, then the l-subgroup $K$ of $G$ that is generated by $H$ is also divisible and abelian.

Proof. If $k \in K$, then $k=\bigvee_{j \in J} \bigwedge_{i \in I} h_{i j}$ with $h_{i j} \in H$ and $J$ and $I$ finite. Thus, for a fixed positive integer $n$ we can find $t_{i j} \in H$ so that $n t_{i j}=$ $h_{i j}$ for all $i \in I$ and $j \in J$, and then $t=\vee \wedge t_{i j} \in K$ and $n t=\vee \wedge n t_{i j}=k$. Thus, $K$ is divisible and abelian.

COROLlary 5.7. The largest divisible subgroup $M$ of an abelian l-group $G$ is an l-subgroup. 
COROLlary 5.8. If $\left\{G_{i} \mid i \in I\right\}$ is a set of divisible abelian l-groups and $G=\bigsqcup G_{i}$ is the abelian l-group free product of the $G_{i}$, then $G$ is divisible.

Proof. The subgroup $H$ of $G$ that is generated by the $G_{i}$ is divisible and $G$ is generated as $l$-group by $H$.

Thus, if we restrict our attention to abelian l-groups, the class of divisible $l$-groups is closed with respect to $l$-homomorphisms, $l$-ideals, joins of $l$ subgroups, cardinal sums and products, and free products. Further, the class of $p$-divisible $l$-groups has these properties (where $G$ is $p$-divisible if $p G=$ $G)$.

Proposition 5.9. For an ordered subfield $F$ of $\mathbb{R}$ the following are equivalent.

(1) $F \cup F$ is archimedean.

(2) $F \cup F$ is an $F$-vector lattice.

(3) If $\left\{G_{i} \mid i \in I\right\}$ is a set of $F$-vector lattices, so is $\sqcup G_{i}$.

(4) If $\left\{G_{i} \mid i \in I\right\}$ is a set of l-subgroups of an abelian l-group $G$ and each $G_{i}$ is an $F$-vector lattice, then so is the l-subgroup of $G$ that is generated by $\sqcup G_{i}$.

(5) $F=\mathbb{Q}$.

Proof. By Corollary 5.4 we have (1) $\Leftrightarrow(5)$ and by the above $(5) \Rightarrow(4)$. Clearly, (4) $\Rightarrow(3) \Rightarrow(2)$. It remains only to show (2) $\Rightarrow(5)$. If $F \supset \mathbb{Q}$

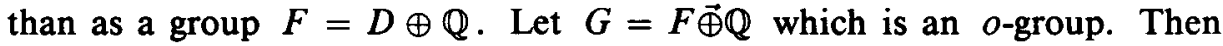
$(d+q, x) \stackrel{\tau}{\longrightarrow}(d+q, q+x)$ is an $o$-automorphism of $G$ and $S=F \times 0 \stackrel{\tau}{\longrightarrow}$ $D(1,0)+\mathbb{Q}(1,1)=T$. Thus, $S$ and $T$ are one-dimensional $F$-vector lattices, but $G=S+T$ is not an $F$-vector lattice. Now, clearly $G$ is an $l$-homomorphic image of $F \sqcup F$ so $F \sqcup F$ is not an $F$-vector lattice.

CoROllary 5.10. If $F \supset \mathbb{Q}$ then an o-group of rank 2 need not contain a largest subgroup that belongs to $V_{F}$.

Corollary 5.11. If $F \neq \mathbb{Q}$ and $G_{1}$ and $G_{2}$ are $F$-vector lattices, then $G_{1} \cup G_{2}$ is not archimedean.

Proof. $F$ is an $l$-subgroup of $G_{1}$ and $G_{2}$ so $F \sqcup F$ is an $l$-subgroup of $G_{1} \sqcup G_{2}$ [12]. Since $F \sqcup F$ is not archimedean neither is $G_{1} \sqcup G_{2}$.

For a subgroup $A$ of $\mathbb{R}$, let $\tilde{A}$ be the torsion class of all normal valued l-groups $G$ where each $G^{\gamma} / G_{\gamma} \cong A$.

Proposition 5.5. $G=A \sqcup A \notin \tilde{A}$. 
Proof. If $G \in \tilde{A}$, then $G^{d}=A^{d} \sqcup A^{d} \in \tilde{A}^{d}$ since $\left(G^{d}\right)^{\gamma} /\left(G^{d}\right)_{\gamma}$ is the divisible hull of $G^{\gamma} / G_{\gamma}$. Thus, it suffices to show that if $A$ is divisible then $A \sqcup A \notin \tilde{A}$.

Case 1. If $A=\mathbb{Q}$, then let $H=\mathbb{Q} \oplus \mathbb{Q} \pi \subseteq \mathbb{R}$ with the natural order. Then $H$ is an $l$-homomorphic image of $G$ but $H \notin \tilde{A}$.

Case 2. If $A \supset \mathbb{Q}$, then $A=D \oplus \mathbb{Q}$ so $H=A \vec{\oplus} \mathbb{Q} \in \tilde{A}$, but it is an $l$-homomorphic image of $G$ (see proof of Proposition 5.9). Therefore, $A \sqcup A \notin \tilde{A}$.

\section{6}

In this section we investigate torsion classes $\tilde{T}$ so that $\tilde{T} \cap V_{F}$ is also a torsion class. Let

$$
\begin{aligned}
\tilde{N}= & \text { torsion class of all normal lest sums of } 0 \text {-groups } \\
= & \text { class of all } l \text {-groups such that the principal polars } \\
& \text { satisfy the DCC. }
\end{aligned}
$$

(See $[5$, p. 3.7] for a proof of the equality of these classes.)

THEOREM 6.1. $V_{F} \cap \tilde{N}$ is a torsion class.

Proof. It suffices to show that each $l$-group $G$ contains a largest convex $l$-subgroup that belongs to $V_{F} \cap \tilde{N}$. Now, such a subgroup must be abelian and divisible. Since the class $\tilde{D}$ of all divisible abelian groups forms a torsion class we may assume that $G \in \tilde{D} \cap \tilde{N}$. But then [9, Theorem 5.1] we may assume that $G=\Sigma\left(\Delta, A_{\delta}\right)$ where $\Delta$ is a root system that satisfies the DCC and each $A_{\delta}$ is a divisible abelian $o$-group. Now each of the $o$-groups $A_{\delta}$ contains a largest convex subgroup $V_{F}\left(A_{\delta}\right)$ that is an $F$-space [8, Proposition 4.2]. Let

$$
\Lambda=\left\{\lambda \in \Delta \mid \delta<\lambda \text { implies } A \in V_{F} \text { and } V_{F}\left(A_{\lambda}\right) \neq 0\right\} .
$$

Then $\Lambda$ is an ideal of $\Delta$ so $H=\Sigma\left(\Lambda, V_{F}\left(A_{\lambda}\right)\right)$ is an $l$-ideal of $G$ that belongs to $V_{F}$.

Now, suppose that $K$ is an $l$-ideal of $G$ that belongs to $V_{F}$ and consider $0<k \in K$ with maximal component $k_{\delta}$. If $\alpha<\delta$, then $G\left(A_{\alpha}\right)=\{g \in G \mid$ each maximal component $g_{\lambda}$ has $\left.\lambda \leq \alpha\right\}$ is an $l$-ideal of $K$ and hence belongs to $V_{F}$. Moreover $A$ is an $l$-homomorphic image of $G\left(A_{\alpha}\right)$ so $A_{\alpha} \in V_{F}$. Similarly, $A_{\delta} \cap K$ must belong to $V_{F}$ so it follows that $K \subset H$. Therefore, $H$ is the torsion kernel of $V_{F} \cap \tilde{N}$ in $G$. 
Note that if $\tilde{K}$ is a torsion class and $\tilde{K} \subseteq \tilde{N}$, then $V_{F} \cap \tilde{K}=V_{F} \cap \tilde{N} \cap \tilde{K}$ is also a torsion class.

CoROllary 6.2. $V_{F} \cap \tilde{F}$ and $V_{F} \cap \tilde{F}_{v} \cap \tilde{D}$ are torsion classes where $\tilde{F}=$ all l-groups such that each bounded disjoint set is finite.

$\tilde{F}_{v}=$ all finite-valued l-groups.

$\tilde{D}=$ all l-groups such that the regular subgroups satisfy the DCC.

THEOREM 6.3. For an abelian l-group $G \in \tilde{N}$ the following are equivalent.

(1) $G \in V_{F}$.

(2) $G / P \in V_{F}$ for each minimal prime $P$.

Proof. $(1 \Rightarrow 2)$ This is obvious.

$(2 \Rightarrow 1)$ Since each $G / P$ is divisible, $G$ is divisible [1]. Without loss of generality let $G=\Sigma\left(\Delta, A_{\delta}\right)$ where $\Delta$ is a root system that satisfies the DCC and each $A_{\delta}$ is a divisible $o$-group. Consider $\delta \in \Delta$ and let $P$ be a minimal prime that does contain $A_{\delta}$. Then $P=A_{\lambda}^{\prime}$ where $\lambda \leq \alpha$ since all minimal primes are of this form. Let $A$ be the sum of all the $A_{\alpha}$ with $\lambda \leq \alpha<\delta$. Then $P+A$ is an $l$-ideal of $G$ and $G /(P+A) \in V_{F}$ since it is a homomorphic image of $G / P$. Now $A_{\delta}$ is $o$-isomorphic to a convex subgroup of $G /(P+A)$ so $A_{\delta} \in V_{F}$. Therefore, $G=\Sigma\left(\Delta, A_{\delta}\right) \in V_{F}$.

REMARK. In [1] there is an example of a hyperarchimedean $l$-group $G$ such that $G / P \cong \mathbb{R}$ for each prime $P$ but $G \notin V_{\mathbb{R}}$.

EXAMPLE 7.1. Let $V=\vec{\prod}_{i=1}^{\infty} \mathbb{R}_{i}$ and let $f$ be an isomorphism of $\mathbb{R}$ onto $\prod_{i=2}^{\infty} \mathbb{R}_{i}$. Then the map $\left(x_{1}, x_{2}, \ldots\right) \stackrel{\tau}{\longrightarrow}\left(x_{1}, x_{2}+f\left(x_{1}\right)_{2}, x_{3}+f\left(x_{1}\right)_{3}, \ldots\right)$ is an $o$-isomorphism of $V$. Let

$$
\begin{aligned}
& A=\left\{\left(x, f(x)_{2}, f(x)_{3}, \ldots\right) \mid x \in \mathbb{R}\right\} \cong \mathbb{R}, \\
& B=\{(x, 0,0,0, \ldots) \mid x \in \mathbb{R}\} \cong \mathbb{R} .
\end{aligned}
$$

Then $A$ and $B$ are archimedean subgroups of $V$ and $A+B=V$.

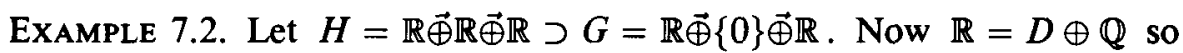
$(d+q, x, y) \stackrel{\tau}{\longrightarrow}(d+q, x+q, y)$ is an $o$-automorphism of $H$. Define $r^{*}(d+q, x, y)=(r((d+q, x, y) \tau)) \tau^{-1}=(r d+r q, r x+r q, r y) \tau^{-1}$. Now, $r d+r q=a+b \in D \oplus \mathbb{Q}$ so $r^{*}(d+q, x, y)=(r d+r q, r x+r q-b, r y)$. Thus, $H$ is an $\mathbb{R}$-hull of $G$ even though $G \in V_{\mathbb{R}}$.

It is easy to extend the preceding construction to get the following result. 
Proposition 7.3. If $G$ is a non-archimedean totally ordered group that belongs to $V_{\mathbb{R}}$ then $G$ admits an $\mathbb{R}$-hull that is a proper extension.

EXAMPLE 7.4. The quotient of two $l$-groups that are not even divisible can be a vector lattice. Let $B$ denote the convex $l$-subgroup of $\prod_{i=1}^{\infty} \mathbb{Z}$ consisting of the bounded sequences of integers. Then $\Pi \mathbb{Z} / B$ is a vector lattice.

Proof. Let $A$ be the convex $l$-subgroup of $\prod_{i=1}^{\infty} \mathbb{R}$ consisting of bounded sequences. Then let

$$
\begin{aligned}
& \Phi: \prod \mathbb{Z} / B \rightarrow \prod \mathbb{R} / A \text { be the obvious } l \text {-homomorphism given by } \\
& \Phi:(x, x, \ldots)+B \rightarrow(x, x, \ldots)+A .
\end{aligned}
$$

Clearly, $\Phi$ is one-to-one. To show that $\Phi$ is onto let $[x]$ denote the largest integer less than or equal to $x$. Then for $\left(x_{1}, x_{2}, \ldots\right) \in \prod \mathbb{R}$ notice that $0 \leq\left(x_{1}, x_{2}, \ldots\right)-\left(\left[x_{1}\right],\left[x_{2}\right], \ldots\right) \leq(1,1, \ldots)$ and hence is in $A$. Thus

$\Phi:\left(\left[x_{1}\right],\left[x_{2}\right], \ldots\right)+B \rightarrow\left(\left[x_{1}\right],\left[x_{2}\right], \ldots\right)+A=\left(x_{1}, x_{2}, \ldots\right)+A$.

Thus $\Pi \mathbb{Z} / B$ is $l$-isomorphic to the vector lattice $\Pi \mathbb{R} / A$. The scalar multiplication is given by $r \cdot\left(\left(n_{1}, n_{2}, \ldots\right)+B\right)=\left(\left[r n_{1}\right],\left[r n_{2}\right], \ldots\right)+B$ for each real number $r$.

EXAMPLE 7.5. The class of vector lattices is not closed with respect to extensions. let $V=\prod_{i=1}^{\infty} \mathbb{R}_{i}$ and let $G=\{v \in V:$ there are real numbers $r_{1}, r_{2}, \ldots, r_{n}$ such that for each $i, v_{i}=\left[v_{i}\right]=r_{j}$ for some $\left.j=1, \ldots, n\right\}$. Let $B$ be the set of bounded sequences in $G$. Then $B$ and $G / B$ are vector lattices but $G$ is not.

Proof. First we show that $G$ is an $l$-subgroup of $V$. Let $x, y \in G$ and let $r_{1}, \ldots, r_{n}$ and $s_{1}, \ldots, s_{m}$ be the real numbers associated with $x$ and $y$, respectively. Then the real numbers $r_{k}-s_{t}$ and $1+\left(r_{k}-s_{t}\right)$ for $k=1, \ldots, n$ and $t=1, \ldots, m$ will suffice for $x-y$. To see this let $x_{i}$ and $y_{i}$ be the $i$ th components of $x$ and $y$. Then by definition of $G$ we have $x_{i}=p+r_{k}$ and $y_{i}=q+s_{t}$ and so $x_{i}-y_{i}=(p-q)+\left(r_{k}-s_{t}\right)$. If $r_{k}-s_{t}>0$ then $\left[x_{i}-y_{i}\right]=p-q$ and so we get $x_{i}-y_{i}-\left[x_{i}-y_{i}\right]=(p-q)+\left(r_{k}-s_{t}\right)-(p-q)=r_{k}-s_{t}$.

$$
\begin{array}{cccc}
p-q-1 & p-q & x_{i}-y_{i} & p-q+1
\end{array}
$$

If $r_{k}-s_{t}<0$ then $\left[x_{i}-y_{i}\right]=p-q-1$ and so we get $x_{i}-y_{i}-\left[x_{i}-y_{i}\right]=$ $(p-q)+\left(r_{k}-s_{t}\right)-(p-q-1)=r_{k}-s_{t}+1$

$$
\begin{array}{llll}
p-q-1 & x_{i}-y_{i} & p-q & p-q+1
\end{array}
$$

Thus $G$ is a group. It is clear that if $x \in G$ then so is $0 \vee x$ and, hence, $G$ is an $l$-subgroup of $V$. 
If $G$ were a vector lattice then it would be a sub-vector lattice of $V$ and hence $\pi \cdot(1,2,3, \ldots)=(\pi, 2 \pi, 3 \pi, \ldots)$ would be in $G$. Thus there would be real numbers $r_{1}, \ldots, r_{k}$ such that for each integer $n, n \pi-[n \pi]=r_{j}$ for some $j$. But then for at least one of the real numbers, say $r_{1}$, we would have $n \pi-[n \pi]=r_{1}=m \pi-[m \pi]$ with $n \neq m$. This says $(n-m) \pi=[n \pi]-[m \pi]$ which is a contradiction. Thus $G$ is not a vector lattice.

Now consider $B$, the set of bounded sequences in $G . B$ is a convex $l$-subgroup of $G$ and is precisely the set of sequences in $G$ that have finite range. To see this let $0<b=\left(b_{1}, b_{2}, \ldots\right) \in B$ and let $b_{i} \leq M$ for all $i$. Let $r_{1}, \ldots, r_{m}$ be the real numbers associated with $b$. Then for each $i, b_{i}=n+r_{j}$ for some integer $n \leq M$ and some $r_{j}, j=1, \ldots, m$. That is, $b$ has finite range. It is also easy to see that any sequences with finite range is in $B$. If a sequence has finite range then so does any scalar multiple of it. Thus $B$ is a sub-vector lattice of $V$.

Finally, $G / B$ is a vector lattice since it is $l$-isomorphic to the vector lattice $V / A$ where $A$ is the bounded sequences in $V$. The isomorphism is $\Phi: G / B \rightarrow V / A$ given by $\Phi(g+B)=(g+A)$ as in the previous example. It is also worth mentioning that $G$ is an $a$-closure of $\prod_{i=1}^{\infty} \mathbb{Z}_{i}$.

The following example was given in [8] as one in which $G^{\gamma} / G_{\gamma} \cong \mathbb{R}$ for all $\gamma \in \Gamma$ but which might not be a vector lattice. We show that it is, in fact, a vector lattice.

EXAMPLE 7.6. $G=\overrightarrow{\sum_{i=1}^{\infty} \mathbb{R} \oplus \mathbb{Q}}(1,1,1, \ldots)$ is a vector lattice.

Proof. Let $\Phi: \sum_{i=1}^{\infty} \mathbb{R} \rightarrow G$ be defined as follows. Choose a basis $\left\{b_{\alpha}\right\}$ for $\mathbb{R}$ over $\mathbb{Q}$ that includes 1 , and let $r \in \mathbb{R}$. Let $r=q+q_{1} b_{\alpha_{1}}+q_{2} b_{\alpha_{2}}+$ $\cdots+q_{n} b_{\alpha_{n}}$ be the unique representation of $r$ as a linear combination of basis elements. Then let

$$
\Phi:(0, \ldots, 0, r, 0, \ldots, 0) \rightarrow(0, \ldots, 0, r, q, q, q, \ldots)
$$

and extend $\Phi$ to all of $\sum_{i=1}^{\infty} \mathbb{R}_{i}$ in the obvious way. It is clear that $\Phi$ is an $o$-isomorphism and it is easy to see that $\Phi$ is onto since

$$
\begin{aligned}
& \Phi:(1,0,0, \ldots) \rightarrow(1,1,1, \ldots) \text { and } \\
& \Phi:(0, \ldots, 0, r,-q, 0, \ldots, 0) \rightarrow(0, \ldots, 0, r, 0, \ldots, 0)
\end{aligned}
$$

Thus $G$ is $o$-isomorphic to $\sum_{i=1}^{\infty} \mathbb{R}_{i}$ and, hence, is a vector lattice.

Furthermore we have $\sum_{i=1}^{\infty} \mathbb{R}_{i} \subseteq \overrightarrow{\prod_{i=1}^{\infty} \mathbb{R}_{i}}$ and so $\Phi$ has a unique extension to an $o$-automorphism of $\prod \mathbb{R}$, call it $\Phi$. Notice that

$$
\Phi(G)=\overrightarrow{\sum_{i=1}^{\infty} \mathbb{R}_{i} \oplus \mathbb{Q}}(1,1,1, \ldots) \oplus \mathbb{Q}(1,2,3, \ldots)
$$


which, by the above, is $o$-isomorphic to $\overrightarrow{\sum_{i=1}^{\infty} \mathbb{R}_{i} \oplus \mathbb{Q}}(1,2,3, \ldots)$ and by an argument similar to the one above this is $o$-isomorphic to $\sum_{i=1}^{\infty} \mathbb{R}_{i}$. The point is that $\Phi(G)$ is a vector lattice. In fact, $G \subset \Phi(G) \subset \Phi^{2}(G) \subset \ldots$ where

$$
\Phi^{n}(G)=\overrightarrow{\sum_{i=1}^{\infty} \mathbb{R}_{i}} \oplus \sum_{i=0}^{i} \mathbb{Q}\left(1^{i}, 2^{i}, 3^{i}, \ldots\right)
$$

and $\Phi^{n}(G)$ is a vector lattice for each $n$. The question is then: Is $\bigcup_{n=0}^{\infty} \Phi^{n}(G)$ a vector lattice? If so, is the scalar multiplication the same as that on $\Phi^{n}(G)$ for each $n$ ? If it is not a vector lattice, then there would be an example of a divisible $o$-group $H$ with $H^{\gamma} / H_{\gamma} \cong \mathbb{R}$ that is not a vector lattice.

\section{8}

We conclude by listing some open questions.

1. Do the vector lattices (over $\mathbb{R}$ ) form a torsion class of $l$-groups?

2. Are any two scalar multiplications on a vector lattice connected by an $l$-automorphism? If not, do $(G, \circ)$ and $(G, \#)$ have the same dimension? In particular is any basis for $\overrightarrow{\sum_{i=1}^{\infty} \mathbb{R}_{i}}$ as a real vector lattice countable?

3. If $G$ is a divisible abelian $o$-group with each $G^{\gamma} / G_{\gamma} \cong \mathbb{R}$, then does $G$ belong to $V_{\mathbf{R}}$ ?

4. If $G$ is an abelian $a^{*}$-closed $l$-group, then does $G$ belong to $V_{\mathbb{R}}$ ? The answer is yes if $G$ is totally ordered or archimedean.

5. If $G$ is an archimedean $l$-group with each $G^{\gamma} / G_{\gamma}$ divisible then is $G$ divisible?

\section{References}

[1] M. Anderson and P. Conrad, 'Epicomplete l-groups', Algebra Universalis 12 (1981), 224-241.

[2] R. Bleier, 'Minimal vector lattice covers', Bull. Austral. Math. Soc. 5 (1971), 331-335.

[3] P. Conrad, J. Harvey, and C. Holland, 'The Hahn embedding theorem for abelian lattice ordered groups', Trans. Amer. Math. Soc. 108 (1963), 143-169.

[4] P. Conrad and J. Diem, 'Polar preserving endomorphisms', Illinois J. Math. 15 (1971), 222-240.

[5] P. Conrad, Lattice ordered groups, (Tulane Lecture Notes, 1970).

[6] P. Conrad, 'Minimal vector lattice covers', Bull. Austral. Math. Soc. 4 (1971), 35-59.

[7] P. Conrad, 'The hulls of representable l-groups and $f$-rings', J. Austral. Math. Soc. 16 (1973), 385-415. 
[8] P. Conrad, 'Changing the scalar multiplication on a vector lattice', J. Austral. Math. Soc. 20 (1975), 332-347.

[9] P. Conrad, The structure of an l-group that is determined by its minimal prime subgroups, pp. 1-21 (Proc. Boise State Conference on Ordered Groups, Marcel Dekker, 1980).

[10] J. Martinez, 'Free products of abelian l-groups', Czechoslovak. Math. J. 23 (1973), 349361.

[11] J. Martinez, 'Torsion theory for lattice ordered groups', Czechoslovak. Math. J. 25 (1975), 284-299.

[12] W. B. Powell and C. Tsinakis, 'Free products in the class of abelian l-groups', Pacific $J$. Math. 104 (1983), 429-442.

Department of Computer Science

Virginia Polytechnic Institute

Blacksburg, Virginia 24061

U.S.A.

Oklahoma State University

Stillwater, Oklahoma 74078

U.S.A.
University of Kansas

Lawrence, Kansas 66044

U.S.A.

Vanderbilt University Nashville, Tennessee 37235

U.S.A. 